\title{
Problemas laborales en pacientes dependientes del alcohol: Evolución en un año de tratamiento.
}

\author{
Work-related problems among alcohol-dependent \\ patients: Development a year of alcohol treatment
}

\author{
$M^{a}$ Trinidad Gómez-Talegón \\ Instituto de Estudios de Alcohol y Drogas \\ Departamento de Farmacología y Terapéutica \\ Facultad de Medicina de Valladolid, España \\ F. Javier Álvarez González \\ Instituto de Estudios de Alcohol y Drogas \\ Departamento de Farmacología y Terapéutica \\ Facultad de Medicina de Valladolid, España \\ Correspondencia: \\ Ma Trinidad Gómez Talegón, \\ Instituto de Estudios de Alcohol y Drogas, \\ Facultad de Medicina, \\ Universidad de Valladolid, \\ 47005 Valladolid, España \\ Tel: + 34983423077 \\ Fax: 983423022 \\ E-mail: trinigt@med.uva.es
}

Resumen

Objetivo: El objetivo de este estudio es analizar la prevalencia de problemas laborales debidos al consumo de alcohol en una muestra de dependientes del alcohol y su evolución durante un año de tratamiento.

Material y Método: Se realizó un estudio prospectivo de una cohorte de 93 pacientes, que realizaron tratamiento durante un año, diagnosticados de dependencia del alcohol según criterios DSM-IV. Se obtuvo información de los siguientes problemas laborales debidos al consumo de alcohol: absentismo, llamadas de atención, sanciones, accidentes laborales, despidos, desempleo o paro, ocurridos en tres momentos: durante toda la vida, el año anterior al inicio del tratamiento y durante un año de tratamiento.

Resultados: El 57\% tuvieron problemas laborales a lo largo de su vida, el 35,5\% el año anterior al inicio del tratamiento, y el 23,7\% durante el año de tratamiento. Los problemas mas frecuentes fueron el absentismo y el desempleo. Los problemas laborales disminuyeron $(\mathrm{p}<0,05)$ durante el año de tratamiento respecto al año anterior al inicio del tratamiento.

Discusión: Este estudio indica que los problemas laborales son frecuentes entre los dependientes del alcohol, y que estos disminuyen con el tratamiento de la dependencia del alcohol.

(Med Segur Trab (Internet) 2009; 55 (216): 20-25)

Palabras clave: Alcohol, Alcoholismo, Problemas relacionados con el alcohol, lugar de trabajo. 


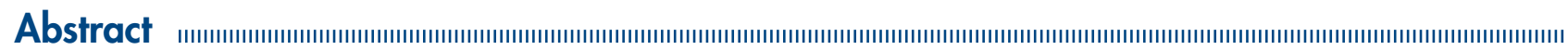

Objetive: The aim of this study is to analyse the prevalence of work-related problems due to the consumption of alcohol in a sample of alcohol-dependent patients and their evolution over twelve months of treatment.

Method: A prospective study was carried out on a cohort of 93 patients diagnosed as alcohol-dependent according to DSM-IV criteria, who underwent treatment over a 12 month period. Information concerning the following work-related problems due to the consumption of alcohol was obtained: absenteeism, warnings, sanctions, accidents at work, dismissals and unemployment, that occurred within three different time scales: the patient's lifetime, the 12 months prior to the start of the treatment and during the 12 months of the treatment.

Results: $57 \%$ had had work-related problems during their lifetime, $35.5 \%$ in the 12 months prior to starting treatment and $23,7 \%$ during the year of treatment. The most frequent problems during the year of treatment were absenteeism and unemployment. Work-related problems decreased $(\mathrm{p}<0.05)$ during the treatment with respect to the year prior to the start of the treatment.

Conclusion: This study indicates that work-related problems are frequent among alcohol-dependent patients, but that such problems decrease when the dependency is treated.

(Med Segur Trab (Internet) 2009; 55 (216): 20-25)

Keywords: Alcohol, Alcoholism, Alcohol-related problems, Workplace. 


\section{INTRODUCCIÓN}

La importancia del alcohol en el medio laboral es indiscutible. Estudios realizados en la población general ${ }^{1,2}$ y en la población trabajadora ${ }^{3-5}$, muestran que los problemas laborales (disminución del rendimiento laboral, absentismo, despido, desempleo o paro, y accidentes laborales) son frecuentes entre los consumidores de alcohol. En algunos estudios se ha analizado la relación de las pautas de consumo de alcohol, y en especial del consumo excesivo de los trabajadores y su relación con estos problemas ${ }^{6-8}$.

Por otra parte se ha señalado que la relación entre consumo de alcohol y problemas laborales es mas compleja de lo que puede parecer ${ }^{9}$. Por una parte estan los factores de riesgo relacionados con consumo de alcohol, tanto laborales (trabajo a destajo, rutinario, insatisfacción, turnos, diponibilidad en el medio laboral, carga de trabajo, etc.) como no laborales (padres consumidores, facilidad para conseguir alcohol, sentirse agobiado, relaciones sociales con bebedores, etc.) ${ }^{10}$. Esto plantea la cuestión de la causalidad invertida: Es decir, si son los factores relativos al puesto de trabajo los que incrementan el consumo de alcohol o no?

Un aspecto sobre el que existe excasa información, es la ocurrencia de problemas laborales entre los trabajadores con dependencia al alcohol, y el posible beneficio de los progamas de prevenciónrealizados por las empresas ${ }^{11}$, o del tratamiento de la dependencia, sobre la ocurrencia de dichos problemas.

Con el fin de analizar estos aspectos se ha diseñado el presente estudio, cuyo objetivo es analizar la prevalencia de problemas laborales relacionados con el consumo de alcohol (durante toda la vida y en el año anterior al inicio del tratamiento), y su evolución durante un año de tratamiento, en una población de pacientes con dependencia al alcohol.

\section{MÉTODOS}

Se diseñó un estudio prospectivo. Los pacientes participantes en dicho estudio fueron los que acudieron a tres centros ambulatorios de tratamiento de alcoholismo de Castilla y León (Spain) en demanda de tratamiento. Todos fueron diagnosticados de dependencia del alcohol siguiendo criterios DSM-IV. El estudio fue aprobado por el Comité Ético de la Facultad de Medicina de Valladolid, y se obtuvo el consentimiento informado de los pacientes participantes.

Se obtuvo información de los siguientes problemas laborales debidos al consumo de alcohol: absentismo laboral, llamadas de atención, sanciones, accidentes laborales, despido, desempleo o paro. Estos problemas laborales fueron evaluados en tres periodos diferentes: i) durante toda la vida, ii) el año anterior al inicio del tratamiento, y iii) durante 12 meses de tratamieto.

Los participantes en el estudio fueron incluidos en un programa de tratamiento cuyo objetivo es la abstinencia del consumo de alcohol. El tratamiento realizado fue mixto: farmacológico, terapias de autoayuda y terapias individuales. Se realizó un seguimiento durante un año de tratamiento, recogiendo la información relativa a los problemas laborales, ocurridos mes a mes durante ese año.

Los datos se analizaron usando el programa estadistico SPSS version 15.0. Se utilizaron las pruebas de Chi cuadrado y T-test para determinar la asociación entre variables, y la prueba de MacNemar para comparar la misma población en dos momentos distintos de tiempo. Valores de $\mathrm{p} \leq 0.05$ fueron considerados estadisticamente significativos.

El estudio incluyó 197 pacientes (165 varones y 32 mujeres) con dependencia al alcohol que iniciaron tratamiento por la misma. De esos 197 pacientes, sólo 93 permanecieron en tratamiento durante 1 año (79 varones y 14 mujeres). El estudio se ha realizado en esos 93 pacientes que han continuado el tratamiento de su dependencia al alcohol durante al menos 
12 meses. La media de edad fue de $44.95 \pm 10.91$ años (media \pm DS), $45.50 \pm 11.30$ entre los varones y $41.85 \pm 7.99$ entre las mujeres $(\mathrm{F}=1.33, \mathrm{p}>0.05)$.

\section{RESULTADOS}

$\mathrm{El}$ 57\% (53 de 93) de los pacientes dependientes tuvieron problemas laborales relacionados con el alcohol durante toda su vida, con una media de $1.86 \pm 1.00$ (media \pm SD) problemas; los mas frecuentes fueron, absentismo 34.4\% y llamadas de atención 28\%. El año anterior al inicio del tratamiento habían tenido problemas el 35.5\% (33 de 93), la media $( \pm$ SD) de problemas fue de $1.87 \pm 1.02$, siendo igualmente los mas frecuentes, absentismo $24.7 \% \mathrm{y}$ llamadas de atención $17.3 \%$.

Durante el año que recibieron tratamiento los pacientes con dependencia al alcohol, tuvieron problemas laborales relacionados con el alcohol el 22.6\% (21 de 93), con una media de problemas de $2.2 \pm 0.98$ (media \pm SD), siendo los mas frecuentes el absentismo $15.1 \%$ y el desempleo $14 \%$.

En ninguno de los tres momentos se han observado diferencias significativas entre sexos ( $p>0.05)$ o rangos de edad (<30 años, 31-40 años, 41-50 años, 51-60 años, 61 a 70 años, $\mathrm{p}>0.05)$.

Durante los 12 meses que permanecieron en tratamiento, la disminución de problemas laborales fue significativa respecto al año antes de iniciar tratamiento $(22.6 \%$ vs $35.5 \%$, $\mathrm{p}<0.05$ en el test de McNemar). Sin embargo, no hay diferencias en la media de problemas laborales el año anterior respecto al año de tratamiento $(t=-1.568, p>0.05)$. Se ha observado un descenso significativo en el absentismo y las llamadas de atención $(\mathrm{p}<0.05$ en el test de McNemar), pero no en los otros tipos de problemas laborales relacionados con el alcohol.

\section{DISCUSIÓN}

En nuestro estudio mas de la mitad de los pacientes con dependecia al alcohol estudiados (57\%), habían tenido problemas laborales relacionados con el consumo de alcohol en algún momento de su vida, una parte importante de esos problemas (35.5\%) se habian concentrado en el año anterior al inicio del tratamiento. Después de un año de tratamiento, la prevalencia de problemas laborales relacionados con el consumo de alcohol descendió de manera significativa, del 35.5\% al 22.6\%.

Los resultados del presente estudio son dificilmente comparables, puesto que la mayor parte de los estudios realizados analizan los problemas laborales en relación con el consumo de alcohol entre la población general y/o población laboral ${ }^{1-8}$, pero no entre los dependientes al alcohol como en el presente estudio. En The Lundby longuitudinal study ${ }^{12}$ una cohorte de la población general de Suecia fue seguida durante varias decadas. Se evaluaron los problemas relacionados con el alcohol entre dependientes del alcohol rehabilitados $(n=12)$ y alcoholicos actuales $(n=11)$. El 18\% de los alcoholicos actuales y por el $8 \%$ de los alcoholicos rehabilitados comunicaron problemas laborales, si bien las diferencias no fueron significativas $(\mathrm{p}=0.59)^{12}$.

Entre nuestros pacientes, y en los tres momentos estudiados, el problema laboral mas frecuente fue el absentismo laboral, seguido de las llamadas de atención y desempleo. El absentismo ya ha sido señalado como una causa frecuente de problema laboral relacionado con el alcohol ${ }^{1}$. Se ha indicado que los bebedores problematicos tienen 2,7 veces mas ausencias del trabajo, relacionadas con enfermedad o accidente, que los bebedores no problematicos (categorizados como tales por el Mortimer-Filkins test) ${ }^{5}$. No obstante la relación entre alcohol y absentismo presenta resultados heterogeneos ${ }^{9}$. El desempleo ha sido frecuentemente referido en relación al consumo de alcohol, si bien la relación entre desempleo y consumo de alcohol es controvertida; asi algunos autores ${ }^{2,6,8}$ han señalado que los bebedores excesivos o no tienen empleo, o es un empleo mas precario. 
La prevalencia de desempleo, y los despidos durante el año de tratamiento no varian en relación al año previo al inicio del tratamiento. Esto pudiera ser debido a: $i$ ) se han registrado con mayor precisión todas las situaciones de desempleo y despido a lo largo del tratamiento, aunque fueran por corto tiempo, $i$ i) algunos pacientes eran desempleados de larga duración que vivian de las ayudas sociales y ya no buscaban empleo, y por lo tanto independientemente del posible efecto del tratamiento seguirían en paro, iii) en algun caso, el despido y desempleo eran consecuencia de situaciones ocurridas antes del inicio del tratamiento (absentismo, baja productividad, etc) y se hizo efectivo cuando el paciente ya habia iniciado su tratamiento, iv) o bien puede ocurrir lo que se ha señalado ${ }^{13}$, de que el exceso de consumo de alcohol entre los parados esta causado mas por el desempleo que viceversa.

Nuestro estudio presenta una serie de limitaciones: $i$ ) los datos han sido recordados, lo que puede dar lugar a minimizaciones, olvidos, ocultaciones, etc., ii) dada la gran tolerancia social que existe en España hacia el alcohol, es posible que algunos pacientes no identifiquen sus problemas laborales en relación con el consumo de alcohol, iii) no todos los pacientes consiguieron la abstinencia al iniciar el tratamiento, por lo que pudieron seguir teniendo problemas laborales hasta conseguirla, iv) en este estudio no se ha analizado aspectos relacionados con el trabajo (ej, rendimiento laboral).

Debemos señalar que en el presente estudio no se ha incluido un grupo control (dependientes del alcohol sin tratamiento), y se han excluido a los pacientes que no han seguido 12 meses de tratamiento por lo que el beneficio obtenido puede estar sobreestimado.

El presente estudio muestra que los problemas laborales son frecuentes entre los pacientes con dependencia al alcohol, así como que se produce un significativo descenso de los mismos con el tratamiento de la dependencia. La evidencia disponible sugiere que los programas preventivos en el lugar de trabajo, para prevenir y reducir los problemas relacionados con el alcohol entre los trabajadores, son efectivos y deberían ser implantados ${ }^{11,14}$. Es por ello que la empresa puede proponer programas preventivos de información o screenig para promover cambios de actitudes sobre el consumo, o intervenir con programas asistenciales que faciliten el tratamiento del trabajador que tiene problemas de dependencia y favorezcan su reinserción laboral y social posteriormente ${ }^{4,14}$.

Tabla I: Prevalencia de problemas laborales relacionados con el consumo de alcohol

\begin{tabular}{lccc}
\hline & $\begin{array}{c}\text { Problemas laborales: } \\
\text { Toda la vida } \\
\text { n (\%) }\end{array}$ & $\begin{array}{c}\text { Problemas laborales: } \\
\text { Año anterior } \\
\text { n (\%) }\end{array}$ & $\begin{array}{c}\text { Problemas laborales: } \\
\text { Año de tratamiento } \\
\text { n (\%) }\end{array}$ \\
\hline Problemas laborales & $53(57)$ & $33(35.5)$ & $21(22.6)$ \\
Ninguno & $32(34.4)$ & $23(24.7)$ & $14(15.1)$ \\
Absentismo & $26(28)$ & $17(18.3)$ & $10(10.8)$ \\
Llamadas de atención & $4(4.3)$ & $2(2.2)$ & 0 \\
Sanciones & $8(8.6)$ & $5(5.4)$ & $1(1.1)$ \\
Accidentes laborales & $16(17.2)$ & $6(6.5)$ & $8(8.6)$ \\
Despido & $15(16.1)$ & $9(9.7)$ & $13(14)$ \\
Desempleo o paro & Toda la vida & $\begin{array}{c}\text { Año anterior } \\
\text { Media } \pm \text { DS }\end{array}$ & Un año de tratamiento \\
& $1.86 \pm 1.0$ & $1.87 \pm 1.02$ & Media \pm DS \\
\hline
\end{tabular}




\section{AGRADECIMIENTOS}

Este estudio ha sido realizado gracias al Convenio específico entre el Ministerio del Interior (Delegación del Gobierno para el Plan Nacional sobre Drogas), la Comunidad Autónoma de Castilla y León (Consejería de Familia e Igualdad de Oportunidades), y a la ayuda para el desarrollo de Redes Temáticas de Investigación Cooperativa, Red de Trastornos Adictivos RD06/0001/0020. Nuestro agradecimiento también a los Centros de tratamiento de alcoholismo A.R - VA (Alcohólicos Rehabilitados de Valladolid) y a los Drs. F. Sánchez y A. Marcos (Cruz Roja de Soria y Valladolid).

\section{BIBLIOGRAFÍA}

1. Jones S. The economic cost of alcohol-related absenteeism and reduced productivity among the working population of New-Zealand. Addiction 1995;90:1455-1461.

2. McDonald Z, Shields MA. Does Problem drinking affect employment? Evidence for England. Health Econ 2004;13:139-55.

3. French MT, Zarkin GA. Is moderate alcohol use related to wages? Evidence from four worksites. J Health Econ. 1995; 14:319-44

4. Mangione TW, Howland J, Amick B, Cote J, Lee M, Bell N, Levine S. Employee drinking practices and work performance. J Stud Alcohol 1999; 60:261-70.

5. Webb GR, Redman S, Hennrikus DJ, Kelman GR, Gibberd RW, Sanson-Fisher RW. The relationships between high-risk and problem drinking and the occurrence of work injuries and related absences. J Stud Alcohol. 1994; 55:434-46.

6. Booth BM, Feng W. The impact of drinking consequences on short-term employment outcomes in at-risk drinkers in six southern states. J Behav Health Serv Res 2002; 29:157-66.

7. Feng W, Zhou W, Butler JS, Booth BM, French MT. The impact of problem drinking on employment. Health Econ. 2001; 10:509-21.

8. Mullahy J, Sindelar J. Employment, unemployment, and problem drinking. J Health Econ 1996;15:409-34.

9. Gmel G, Rehm J. Harmful alcohol use.Alcohol Res Health. 2003; 27:52-62.

10. Ochoa Mangado E, Madoz Gúrpide A. Consumo de alcohol y otras drogas en el medio laboral. Med Segur Trab 2008 54: 25-32

11. Gómez-Talegón MT, Alvarez Gonzalez FJ. Alcohol y prevención de los problemas relacionados con el consumo de alcohol en el ambito laboral. Med Segur Trab 2005; 198: 65-72.

12. Ojesjo L. The recovery from alcohol problems over the life course: the Lundby longitudinal study, Sweden. Alcohol. 2000; 22:1-5.

13. Forcier MW. Unemployment and alcohol abuse: a review. J Occup Med. 1988; 30:246-51.

14. Roman PM, Blum TC. The Workplace and Alcohol Problem Prevention. Alcohol Res Health 2002; 26:49-57. 